\title{
Hepatocellular Oxidant Stress following Intestinal Ischemia-Reperfusion Injury ${ }^{1,2}$
}

\author{
R. H. Turnage, J. Bagnasco, J. Berger, K. S. Guice, K. T. Oldham," and D. B. Hinshaw \\ Sections of General Surgery and *Pediatric Surgery, Department of Surgery, University of Michigan Medical School, Ann Arbor, Michigan 48109
}

Submitted for publication November 20, 1990

Reperfusion of ischemic intestine results in acute liver dysfunction characterized by hepatocellular enzyme release into plasma, reduction in bile flow rate, and neutrophil sequestration within the liver. The pathophysiology underlying this acute hepatic injury is unknown. This study was undertaken to determine whether oxidants are associated with the hepatic injury and to determine the relative value of several indirect methods of assessing oxidant exposure in vivo. Rats were subjected to a standardized intestinal ischemia-reperfusion injury. Hepatic tissue was assayed for lipid peroxidation products and oxidized and reduced glutathione. There was no change in hepatic tissue total glutathione following intestinal ischemia-reperfusion injury. Oxidized glutathione (GSSG) increased significantly following 30 and $60 \mathrm{~min}$ of reperfusion. There was no increase in any of the produets of lipid peroxidation associated with this injury. An increase in GSSG within hepatic tissue during intestinal reperfusion suggests exposure of hepatocytes to an oxidant stress. The lack of a significant increase in products of lipid peroxidation suggests that the oxidant stress is of insufficient magnitude to result in irreversible injury to hepatocyte cell membranes. These data also suggest that the measurement of tissue GSSG may be a more sensitive indicator of oxidant stress than measurement of products of lipid peroxidation. 1991 Academic Press, Inc.

\section{INTRODUCTION}

Reperfusion of ischemic intestine in the rat is associated with acute injury of the lung, liver, and kidney [1, 2]. Understanding the mechanisms responsible for the remote organ injury in this model may provide insight into the pathophysiology of the syndrome of multiple organ failure in critically ill patients. In particular, he-

\footnotetext{
${ }^{1}$ Presented at the Annual Meeting of the Association for Academic Surgery, Houston, TX, November 14-17, 1990.

${ }^{2}$ Supported in part by NIH Grants HL38141 and GM41745 and a Veterans Administration Merit Review grant.
}

patic dysfunction often presages death in patients with multiple organ failure and is the subject of this investigation.

Acute hepatic injury following intestinal ischemia-reperfusion is characterized by an acute rise in serum levels of hepatocellular enzymes, a reduction in bile flow rate, and sequestration of neutrophils within the hepatic parenchyma $[2,3]$. The pathophysiology of the acute liver injury is unclear; however, the prominent neutrophil sequestration and data from other related injury models suggest that potentially important mediators of both local and remote organ injury may be oxygen radical species. Oxidants have been incriminated in a variety of experimental hepatic injuries, including those associated with ischemia [4], sepsis [5], endotoxemia [6], and exposure to hepatocellular toxins such as alcohol [7] and carbon tetrachloride [8].

It is clear that oxygen radicals are involved in the reperfusion injury of ischemic intestine [9]. Whether these oxidants directly or indirectly participate in the pathogenesis of the associated hepatic injury is unknown. The purpose of this investigation was to evaluate the role of oxidants in the acute hepatic injury which follows intestinal ischemia-reperfusion. The data support the concept that oxidant stress occurs in the liver and is correlated temporally with parameters of hepatic injury such as reduction in bile flow and the release of hepatocellular enzymes into the plasma. In addition, a differential assessment is provided for several biochemical markers which are used to provide indirect evidence of in vivo oxidant exposure.

\section{MATERIALS AND METHODS}

\section{Animal Model}

All protocols were approved by the University of Michigan Committee on Use and Care of Animals in accordance with existing National Institutes of Health guidelines. Male Sprague-Dawley rats $(150 \mathrm{~g})$ underwent laparotomy following ketamine $\mathrm{HCl}$ anesthesia $(100 \mathrm{mg} / \mathrm{kg}$ ip). A noncrushing microvascular clip was placed across the proximal superior mesenteric artery and the cessa- 
tion of arterial pulsation within the vasa recta noted. Prior experiments in our laboratory have demonstrated an $85 \%$ reduction in mucosal blood flow following this maneuver [1]. After 60 or $120 \mathrm{~min}$ of ischemia, the clip was removed with restoration of mucosal blood flow to approximately $85 \%$ of preocclusion levels [1]. Following 0,30 , and 60 min of intestinal reperfusion, the left lateral segment of the liver was excised and half was frozen immediately in liquid nitrogen and half was placed on ice. The frozen specimen was maintained at $-70^{\circ} \mathrm{C}$ until assay for glutathione. The fresh specimens were maintained on ice and assayed for products of lipid peroxidation within $3 \mathrm{hr}$ of harvesting (previous experiments in our laboratory have demonstrated that cold, fresh tissue is required for reproducible results with these assays). Time-matched control animals underwent laparotomy with exposure of the superior mesenteric artery without the placement of a microvascular clip. These shamoperated animals were otherwise prepared identically with the experimental animals.

\section{Assays}

The assays for products of lipid peroxidation were performed on fresh liver tissue which was homogenized and sonicated prior to assay [10].

\section{Conjugated Dienes (CD)}

The assay for CD is previously described [10] and involves the spectrophotometric determination of increased uv absorption in the hepatic tissue samples at $235 \mathrm{~nm}$. This correlates with the formation of radical intermediates from fatty acids with configuration into a conjugated diene structure which absorbs at this wavelength [11].

\section{Fluorescent Products (FP)}

This assay for fluorochromic proteins with features of Schiff bases is previously described [10]. Briefly, the assay involves the extraction of fluorescent products from the liver tissue with a chloroform-methanol (Folch) extraction. Following the addition of water, the methanol$\mathrm{H}_{2} \mathrm{O}$ fraction is analyzed for fluorescence at $430 \mathrm{~nm}$ when excited at $360 \mathrm{~nm}$. The results are standardized with $1 \mu \mathrm{g} / \mathrm{ml}$ quinine sulfate [10].

\section{Malondialdehyde (MDA)}

The thiobarbituric acid activity assay was used for the quantitive estimation of malondialdehyde. Briefly, a 1:1:1 solution of trichloroacetic acid, thiobarbituric acid, and $\mathrm{HCl}$ was added to homogenized tissue. The solution was heated to $100^{\circ} \mathrm{C}$ and the absorbance of the supernatant was determined at $535 \mathrm{~nm}$. Butylated hydroxytoluene was added to prevent further lipid peroxidation during the assay [10].

\section{Glutathione}

Total glutathione (GSH) and oxidized glutathione (GSSG) were determined as previously reported [12]. These assays involve the spectrophotometric monitoring of the rate of formation of 2-nitro-5-thiobenzoic acid by the sequential oxidation of 5,5-dithio-bis-( 2 nitrobenzoic acid) and its reduction by NADPH in the presence of glutathione reductase.

\section{Statistical Analysis}

Data were evaluated by a one-way analysis of variance (ANOVA) with a post hoc comparison among groups using either Tukey's method for comparison of lipid peroxidation products or Fisher's protected least squares difference (PLSD) method for GSH and GSSG. Statistical significance was assigned when $P<0.05$. A minimum of five animals are included in each experimental and control group and the data and statistical analysis are shown for each individual experiment. All data are expressed as the means \pm standard error of the mean.

\section{RESULTS}

\section{Products of Lipid Peroxidation}

Conjugated dienes (Table 1). Hepatic tissue levels of conjugated dienes were assessed following $120 \mathrm{~min}$ of ischemia (without reperfusion) and following 10,30, and 60 min of reperfusion. At none of these time points were hepatic tissue levels of conjugated dienes significantly elevated above those of sham-operated control animals.

Fluorescent products (Table 1). Hepatic tissue levels of fluorescent products were assessed following $120 \mathrm{~min}$ of ischemia (without reperfusion) and following 10, 30, and $60 \mathrm{~min}$ of reperfusion. The results of the ANOVA indicate that the differences among these groups are marginal but not significant ( $P=0.0634$ ). Post hoc comparison using Tukey's method indicates that the fluorescence in the 120/10 group is lower than in the $120 / 60$ group $(P<0.05)$. A single difference of this sort between two groups is consistent with the fact that the differences for the whole series of experiments are marginal. It is for this reason that the number of observations for fluorescent products is higher. However, statistically meaningful differences were not discernible even with larger study groups. Of the conventional analyses for lipid peroxidation products in this model, only this single comparison achieved a $P$ value less than 0.05 .

Malondialdehyde (Table 1). Liver tissue levels of malondialdehyde were not elevated following intestinal ischemia reperfusion.

\section{Hepatic Total Glutathione and Oxidized Glutathione}

Total glutathione (Fig. 1). Total glutathione levels within the liver tissue remained unchanged in experimental animals when compared to sham-operated con- 
TABLE 1

Products of Lipid Peroxidation in Liver Tissue following Intestinal Ischemia-Reperfusion Injury

\begin{tabular}{cccc}
$\begin{array}{c}\text { Group } \\
\text { (ischemia/reperfusion) }\end{array}$ & $\begin{array}{c}\text { MDA } \\
(535 \mathrm{~nm})(n)\end{array}$ & $\begin{array}{c}\text { CD } \\
(233 \mathrm{~nm})(n)\end{array}$ & $\begin{array}{c}\text { FP } \\
(430 \mathrm{~nm})(n)\end{array}$ \\
\hline $0 / 180$ (sham) & $0.12 \pm 0.02(10)$ & $3.6 \pm 0.4(9)$ & $20.3 \pm 2.3(17)$ \\
$120 / 00$ & $0.09 \pm 0.02(11)$ & $2.0 \pm 0.3(8)$ & $14.3 \pm 1.6(12)$ \\
$120 / 10$ & $0.07 \pm 0.1(9)$ & $2.7 \pm 0.5(6)$ & $16.5 \pm 2.3(15)$ \\
$120 / 30$ & $0.06 \pm 0.01(5)$ & $2.4 \pm 0.6(5)$ & $18.1 \pm 1.2(10)$ \\
$120 / 60$ & $0.09 \pm 0.02(9)$ & $2.7 \pm 0.5(7)$ & $21.4 \pm 2.9(2)$ \\
\hline
\end{tabular}

Note. Data are expressed as absorbance at the indicated wave length. ( $n$ ), Number of observations; MDA, malondialdehyde; CD, conjugated dienes; FP, fluorescent products. Ischemia/reperfusion designated as 120/30 represents 120 min of ischemia followed by 30 min of reperfusion, etc.

${ }^{*} P<0.05$; see text.

trols, regardless of the duration of intestinal ischemiareperfusion injury. This was true following $60 \mathrm{~min}$ of ischemia as well (data not shown).

Oxidized glutathione (Figs. 2 and 3). Hepatic parenchymal oxidized glutathione levels are expressed quantitatively in moles $/ 50 \mathrm{mg}$ of tissue (Fig. 2) and as the ratio of GSSG to GSH (Fig. 3). The latter format is in order to facilitate comparison with other reports. Intestinal ischemia-reperfusion injury resulted in a significant increase in the concentration of oxidized glutathione within the liver, expressed both in absolute concentrations (Fig. 2) and as the fraction of total glutathione present in oxidized form (Fig. 3). Thirty minutes following the reperfusion of ischemic intestine (120/30), tissue GSSG increased more than threefold compared to control levels $\left(3.6 \pm 1.19 \times 1910^{-9}\right.$ vs $1.01 \pm 0.38 \times 10^{-9}$

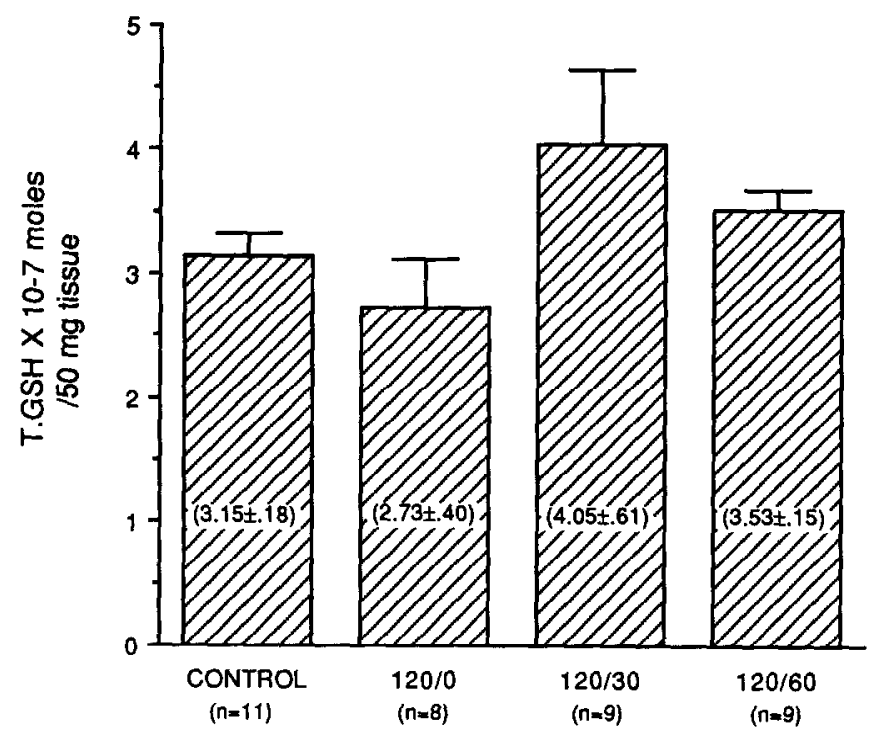

FIG. 1. Hepatic GSH following intestinal ischemia-reperfusion injury. There is no significant difference in GSH levels in liver tissue between control and experimental groups $(P>0.05$, ANOVA, post hoc comparison using Fisher's PLSD method, $n=$ number of observations). moles $/ 50 \mathrm{mg}$ tissue, $P<0.05$ as shown). This significant increase in GSSG persisted to $60 \mathrm{~min}$ of reperfusion $\left(3.97 \pm 0.7 \times 10^{-9}\right.$ vs $1.54 \pm 0.57 \times 10^{-9}$ moles $/ 50 \mathrm{mg}$ tissue, $120 / 60$ vs $120 / 0, P<0.05$ as shown). The proportion of GSSG increased more than fourfold (from $0.3 \pm$ $0.1 \%$ in control animals to $1.4 \pm 0.3 \%$ in the $120 / 30$ group, $P<0.05$ ). The percentage of GSSG remained significantly elevated following $60 \mathrm{~min}$ of reperfusion when compared to sham-operated control animals. In the absence of reperfusion, intestinal ischemia was not associated with a significant change in oxidized glutathione levels.

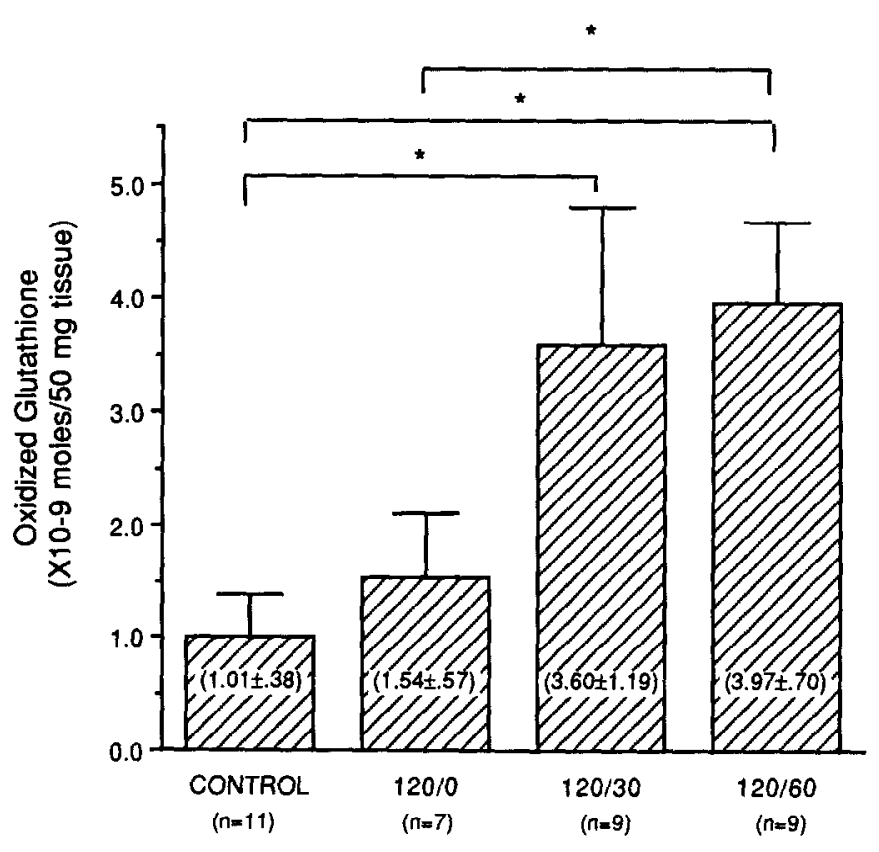

FIG. 2. Hepatic tissue GSSG following intestinal ischemia-reperfusion injury. Intestinal reperfusion was associated with a significant increase in GSSG over sham-operated control animals. In addition, animals with $120 \mathrm{~min}$ of ischemia and $60 \mathrm{~min}$ of reperfusion $(120 / 60)$ had significant GSSG elevations compared to the group with ischemia alone $(120 / 0) .\left({ }^{*} P<0.05\right.$, ANOVA with post hoc comparison using Fisher's PLSD method, $n=$ number of observations). 


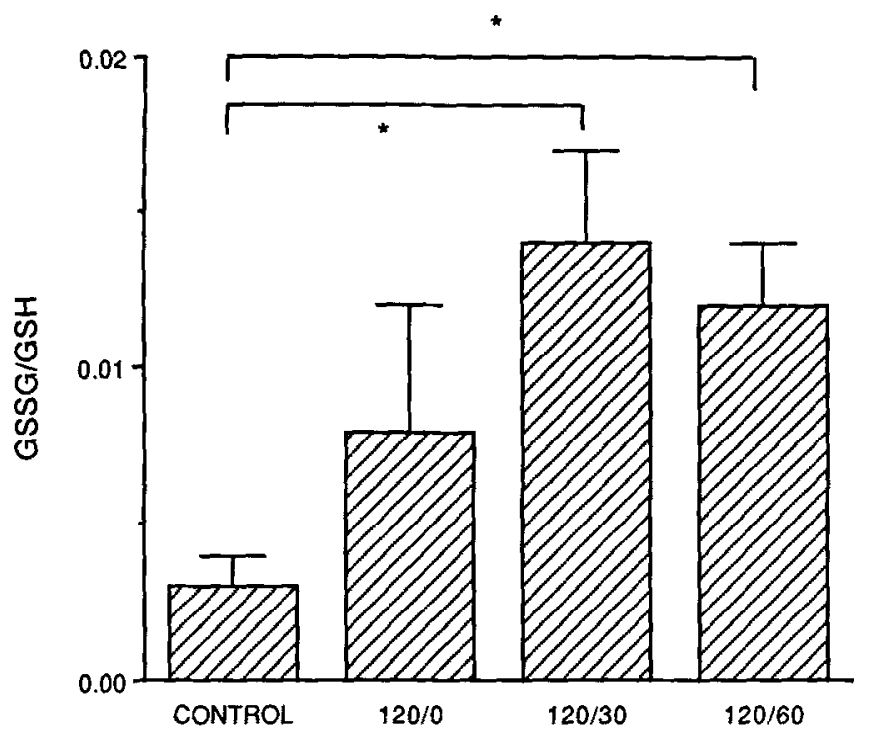

FIG. 3. Ratio of GSSG to total GSH within hepatic tissue following intestinal ischemia-reperfusion injury. Intestinal reperfusion was associated with a significant (threefold) increase in the oxidized fraction of total glutathione within the liver although the fraction of glutathione in the GSSG form is only 1 to $2 \%$ of the total $\left(^{*} P<0.05\right.$, ANOVA with post hoc comparison using Fisher's PLSD method, $n=$ number of observations).

Periods of sublethal intestinal ischemia (less than 120 $\mathrm{min}$ ) [13] were associated with a significant increase in oxidized glutathione only following prolonged periods of reperfusion (Fig. 4). Following $60 \mathrm{~min}$ of intestinal ischemia, the amount of oxidized glutathione remained unchanged compared to controls until 120 min of reperfusion, at which time liver GSSG increased to 3.3 times control values $\left(3.34 \pm 1.2 \times 10^{-9}\right.$ vs $1.01 \pm .38 \times 10^{-9}$ moles $/ 50 \mathrm{~g}$ tissue, $60 / 120 \mathrm{vs}$ control, $P<0.05$, as shown).

\section{DISCUSSION}

Intestinal ischemia-reperfusion injury is associated with remote organ dysfunction. The hepatic injury is characterized by a reduction in bile flow rate, release of hepatocellular enzymes into the plasma, and neutrophil infiltration as noted $[2,3]$. A variety of mediators including oxidants appear to be involved. Data consistent with this are provided by Keller et al. [5] who demonstrated a significant decrease in hepatic tissue GSH levels in experimental septic shock and suggested that sepsis-induced oxidant stress depleted hepatic GSH reserves and potentially rendered the liver more susceptible to additional oxidant exposure. The purpose of this investigation was twofold: (1) to assess whether oxidant stress occurs in the liver following intestinal ischemia-reperfusion injury and (2) to compare the sensitivity of lipid peroxidation products and oxidized glutathione in tissue as markers for in vivo oxidant exposure.
Oxygen radicals react with the unsaturated fatty acid moieties of cellular membranes via the process of lipid peroxidation. A variety of toxic (organic peroxides) and nontoxic products are produced through amplification of this reaction. The degree of lipid peroxidation of cellular membranes may be indirectly assessed by measuring tissue or plasma conjugated dienes, fluorescent products, and malondialdehyde [10]. Despite sampling tissue at a variety of time points during intestinal ischemia-reperfusion injury, no significant increases in any of these markers of lipid peroxidation were noted in these experiments.

GSH is a ubiquitous intracellular tripeptide which is particularly abundant within the liver. It appears to be a principal endogenous antioxidant, providing reducing capacity for a variety of reactions that detoxify organic peroxides, $\mathrm{H}_{2} \mathrm{O}_{2}$, and other oxygen radicals. Reduced glutathione normally constitutes $99 \%$ or more of the total glutathione present. Upon oxidant exposure, glutathione peroxidase catalyzes the oxidation of GSH to form GSSG according to the following equation $[12,14]$ :

$$
2 \mathrm{GSH}+\mathrm{H}_{2} \mathrm{O}_{2} \underset{\text { GSSG reductase }}{\stackrel{\text { GSH peroxidase }}{\rightleftarrows}} \mathrm{GSSG}+2 \mathrm{H}_{2} \mathrm{O}
$$

Thus assessment of GSSG provides a potential marker for oxidant stress in vivo [12].

After $120 \mathrm{~min}$ of intestinal ischemia and $30 \mathrm{~min}$ of reperfusion, hepatic tissue GSSG increased threefold. This increase persisted through the $60-\mathrm{min}$ period of re-

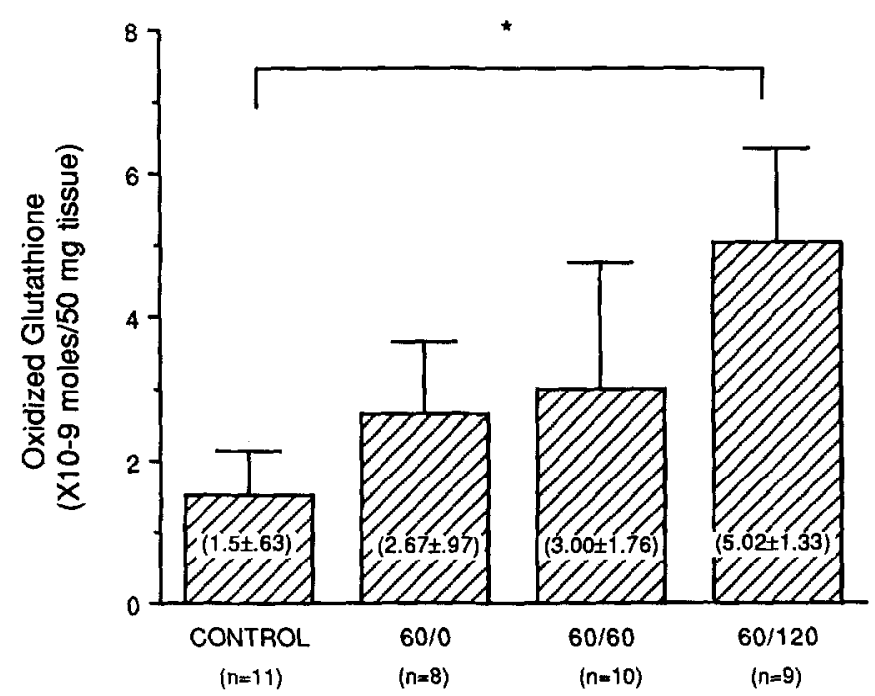

FIG. 4. Hepatic tissue GSSG following $60 \mathrm{~min}$ of ischemia and reperfusion up to $120 \mathrm{~min}$. A significant increase in hepatic GSSG was seen following $120 \mathrm{~min}$ of reperfusion. Lesser periods of reperfusion were not associated with a significant increase in hepatic tissue GSSG $\left({ }^{*} P<0.05\right.$, ANOVA with post hoc comparison using Fisher's PLSD method, $n=$ number of observations). 
perfusion. Ischemia without reperfusion was not associated with a significant increase in hepatic GSSG. These data suggest that an oxidant stress occurs within the liver following reperfusion of ischemic intestine. Unlike the septic shock model of Keller et al. [5], total GSH levels were unchanged in this model of intestinal ischemia-reperfusion injury. The absence of change in total GSH levels has several possible explanations: (1) because only a small fraction ( 1 to $2 \%$ ) of GSH is oxidized to GSSG, the threshold is too low for detection when only total GSH is analyzed, (2) that recycling via the glutathione reductase pathway occurs very rapidly, or (3) alternatively, that GSSG derived from the intestine has been delivered to the liver in portal venous plasma and that the GSH and GSSG content of the liver itself is unchanged. The latter possibility appears least likely after semiquantitative extrapolations from previous data [12]. The former and more likely possibilities are consistent with the hypothesis that the liver injury following intestinal ischemia-reperfusion is associated with an oxidant stress of lesser magnitude than that produced by Keller's sepsis model.

The absence of a significant increase in tissue products of lipid peroxidation suggests several possibilities. First, that the oxidant stress to which the liver is exposed is not of sufficient magnitude to result in peroxidation of lipid membranes. This is consistent with other data suggesting that the liver injury is one of potentially reversible hepatocyte dysfunction rather than cell death $[2,3,13]$. Alternatively, it is possible that lipid peroxidation does occur, but that endogenous hepatocyte antioxidant systems limit the process to marginal or undetectable levels. It is possible, indeed probable, that hepatocyte dysfunction following intestinal ischemiareperfusion involves mechanisms other than, or in addition to, oxidant exposure. Perhaps the most important observation in this study is that GSSG tissue levels appear to be a more sensitive marker of oxidant stress than lipid peroxidation products (CD, MDA, FP). The potential usefulness of this marker is enhanced if it is indeed sensitive enough to detect potentially reversible injury rather than cell death.

In summary, it appears that intestinal ischemia-reperfusion is associated with hepatocellular injury resulting in the oxidation of glutathione without significant total glutathione depletion. This suggests, but does not prove, that hepatocytes are exposed to oxidant stress following intestinal reperfusion. The absence of an increase in lipid peroxidation products in hepatic tissue suggests that this oxidant exposure produces insufficient lipid peroxidation to be detected by commonly employed assays for CD, FP, and MDA. The fact that oxidized glutathione levels are elevated suggests that GSSG determination may be a more sensitive indicator of oxidant exposure.

\section{REFERENCES}

1. Schmeling, D. J., Caty, M. G., Oldham, K. T., Guice, K. S., and Hinshaw, D. B. Evidence for neutrophil-related acute lung injury after intestinal ischemia-reperfusion. Surgery 106: 195, 1989.

2. Turnage, R. H., Oldham, K. T., Guice, K. S., Hinshaw, D. B., and Weatherbee, $\mathrm{L}$. Hepatic injury following intestinal ischemia-reperfusion. FASEB 4(3): A761, 1990. [Abstract]

3. Turnage, R. H., Guice, K. S., Oldham, K. T., Bagnasco, J., and Weatherbee, L. Evidence for a neutrophil-related acute liver injury following intestinal ischemia-reperfusion. Circ. Shock 31: 42, 1990. [Abstract]

4. Marubayashi, S., Dohi, K., Ochi, K., and Kawasaki, T. Role of free radicals in ischemic rat liver cell injury: Prevention of damage by alpha-tocopherol administration. Surgery 99: 184, 1986.

5. Keller, G. A., Burke, R., Harty, V. T., Humphrey, E., and Surinam, R. L. Decreased hepatic glutathione levels in septic shock: Predisposition of hepatocyte to oxidative stress. Arch. Surg. 120: $941,1985$.

6. Ogawa, R., Morita, T., Kunimoto, F., and Fujita, T. Changes in hepatic lipoperoxide concentration in endotoxic rats. Circ. Shock 9: $369,1982$.

7. Sinaceur, J., Ribiere, C., Sabourault, D., and Nordman, R. Superoxide formation in liver mitochondria during ethanol intoxication: Possible role in alcohol hepatotoxicity. In G. Poli, K. Cheeseman, M. Dianzani, and T. Slater (Eds.), Free Radicals in Liver Injury. Oxford: IRL Press, 1985.

8. Dianzani, M. U., and Poli, G. Lipid peroxidation and haloalkylation in $\mathrm{CCl}_{4}$-induced liver injury. In G. Poli, K. Cheeseman, $\mathrm{M}$. Dianzani, and T. Slater (Eds.), Free Radicals in Liver Injury. Oxford: IRL Press, 1985.

9. Parks, D. A., and Granger, D. N. Ischemia-induced vascular changes: Role of xanthine oxidase and hydroxyl radical. $A m$. J. Physiol. 245: G285, 1983.

10. Ward, P. A., Till, G. O., Hatherill, J. R., Annesley, T. M., and Kunkel, R. G. Systemic complement activation, lung injury, and products of lipid peroxidation. J. Clin. Invest. 76: 517, 1985.

11. Klein, R. A. The detection of oxidation in liposome preparations. Biochem. Biophys. Acta 210: 486, 1970.

12. Abdulla, E. K., Caty, M. G., Guice, K. S., Hinshaw, D. B., and Oldham, K. T. Arterial levels of oxidized glutathione (GSSG) reflect oxidant stress in vivo. J. Surg. Res. 48: 291, 1990.

13. Gerkin, T., Turnage, R. H., Guice, K. S., and Oldham, K. T. Pulmonary endothelial cytotoxicity following lethal and sublethal intestinal ischemia-reperfusion injury (in press).

14. Meister, A. Glutathione. In I. Arias, W. Jakoby, H. Popper, D. Schachter, and D. Shafritz (Eds.), The Liver: Biology and Pathobiology. New York: Raven Press, 1988. 